\title{
The Aggregation of Brucella abortus Occurs Under Microaerobic Condi- tions and Promotes Desiccation Tolerance and Biofilm Formation
}

\author{
Marta A. Almirón ${ }^{1, *}$, Mara S. Roset ${ }^{1}$ and Norberto Sanjuan ${ }^{2}$ \\ ${ }^{1}$ Instituto de Investigaciones Biotecnológicas (IIB-INTECH), Consejo Nacional de Investigaciones Científicas y Técni- \\ cas, Universidad Nacional de San Martín, Buenos Aires, Argentina \\ ${ }^{2}$ Laboratorio de Microscopía Electrónica y Patología Experimental, Departamento de Microbiología, Parasitología e \\ Inmunología. Facultad de Medicina, Universidad de Buenos Aires, Buenos Aires, Argentina
}

\begin{abstract}
Brucella abortus causes brucellosis mainly in cattle. The infection is transmitted to humans by ingestion of animal products or direct contact with infected material. While the intracellular lifestyle of Brucella is well characterized, its extracellular survival is poorly understood. In nature, bacterial persistence is associated with biofilms, where aggregated cells are protected from adversity. The inability of Brucella abortus to aggregate under aerobiosis and that fact that the replicative niche of Brucella is characterized by microaerobic conditions prompted us to investigate the capacity of this pathogen to aggregate and grow in biofilms under microaerobiotic conditions. The results show that B. abortus aggregates and produces biofilms. The aggregates tolerate desiccation better than planktonic cells do, adhere and displace even in the absence of the lipopolysaccharide-O antigen, flagella, the transcriptional regulator VjbR, or the enzymes that synthesize, transport, and modify cyclic $\beta(1,2)$ glucan.
\end{abstract}

Keywords: Aggregation, biofilms, Brucella, desiccation, flotation, VjbR.

\section{INTRODUCTION}

Infection with Brucella can result in brucellosis, a chronic lifelong zoonotic disease in humans and cattle. This is a damaging disease, particularly in developing countries with economies that depend on livestock. In humans, transmission occurs mainly through direct contact with infected animals or ingestion of contaminated dairy products, while in wildlife, it is spread due to bacterial persistence in the environment. Although Brucella spp. can survive for long periods in soil, dust, slurry, water, and meat, the survival strategies of this pathogen have been mostly studied from within eukaryotic cells.

Bacteria that colonize different surfaces and invade susceptible hosts to cause chronic infections grow predominantly in biofilms $[1,2]$. First, bacteria produce an extracellular matrix mainly composed of exopolysaccharides, which surrounds them and plays a key role in keeping them hydrated and helping them adhere to inert or biological surfaces. Even if adhesion is not a population-dependent phenotype [3], it is necessary for the complex process of biofilm growth. Experimental conditions of wild-type Brucella growth, as a stable community of cells that form surfaceadherent biofilms, have not been demonstrated. Adhesion has been reported only in mutants of $B$. melitensis that present an altered expression of outer-membrane proteins [4-6].

*Address correspondence to this author at the UNSAM- Campus MigueleteIIB, Av. 25 de Mayo y Francia, (1650) San Martín, Buenos Aires, Argentina Tel: (5411) 4006 1500; Fax: (5411) 4006 1559;

E-mail: malmiron@iib.unsam.edu.ar
One of these mutants is $B$. melitensis vjbR, which cannot express the transcriptional regulator $\mathrm{VjbR}$. As a $B$. melitensis double mutant $v j b R$ omp31 did not aggregate under aerobic conditions, it was postulated that Omp31 is involved in the production and/or secretion of the matrix-forming exopolysaccharides. To support this hypothesis Uzureau et al. demonstrated that similar mutations in B. abortus vjbR do not cause aggregation under the same conditions due to the natural absence of omp31 in this species [4].

Given the fact that Brucella usually survives in niches characterized by low oxygen tension and nutritional scarcity $[7,8]$ in infected hosts or in the environment and secretes $\mathrm{N}$ dodecanoyl homoserine lactone, which (along with VjbR) belongs to the quorum-sensing system [9-11], we hypothesized that wild-type B. abortus produces an extracellular matrix that confers adhesion and stress-resistance properties and promotes the development of biofilms under nutritionally deficient, microaerobic conditions.

\section{Autoaggregation of $\boldsymbol{B}$. Abortus}

We initiated our work by studying the physiology of $B$. abortus under microaerobic conditions. First, oxygen preference in wild-type B. abortus 2308 was assayed in semi-solid media containing thioglycolate. This bacterium behaved as a microaerophile; it produced a zone of growth a few millimetres below the surface of the medium after 48 hours of incubation in a glass tube. However, with prolonged incubation beginning at day 3 to $5, B$. abortus produced a discontinuous turbidity growing downward towards the bottom of the tube. Though growth was observed in the centre of the tube, it was 
prominent along the walls as a film growing downward but unattached to the glass wall (Fig. 1A).

Therefore, we looked for biofilm development in Brucella broth (BB) cultures in glass tubes under microaerobiosis. The microaerobic environment was generated by a $5 \% \mathrm{CO} 2$ incubator or in $8 \mathrm{~mL}$ culture media in $10-\mathrm{mL}$ screw-capped tubes, incubated statically at $37^{\circ} \mathrm{C}$. Under these conditions, bacterial growth first occurred at the bottom of the tubes, consistent with its non-motile phenotype. Interestingly, the cellular mass became viscous during incubation. From day 4 to day 7 in static cultures at $37^{\circ} \mathrm{C}$, threads spontaneously detached from the viscous bacterial mass were hanging from one end attached to the glass at the airliquid interface. After 2 weeks, a discontinuous ring appeared and the threads had grown longer (Fig. 1B). This behaviour was not apparent when similar cultures were incubated with shaking for the same period of time to maintain aerobic conditions. The attachment to the glass at the airliquid interface was weak; the threads easily fell down and the rings were washed away when adhesion was examined by Crystal violet staining [12]. As a result, no confident

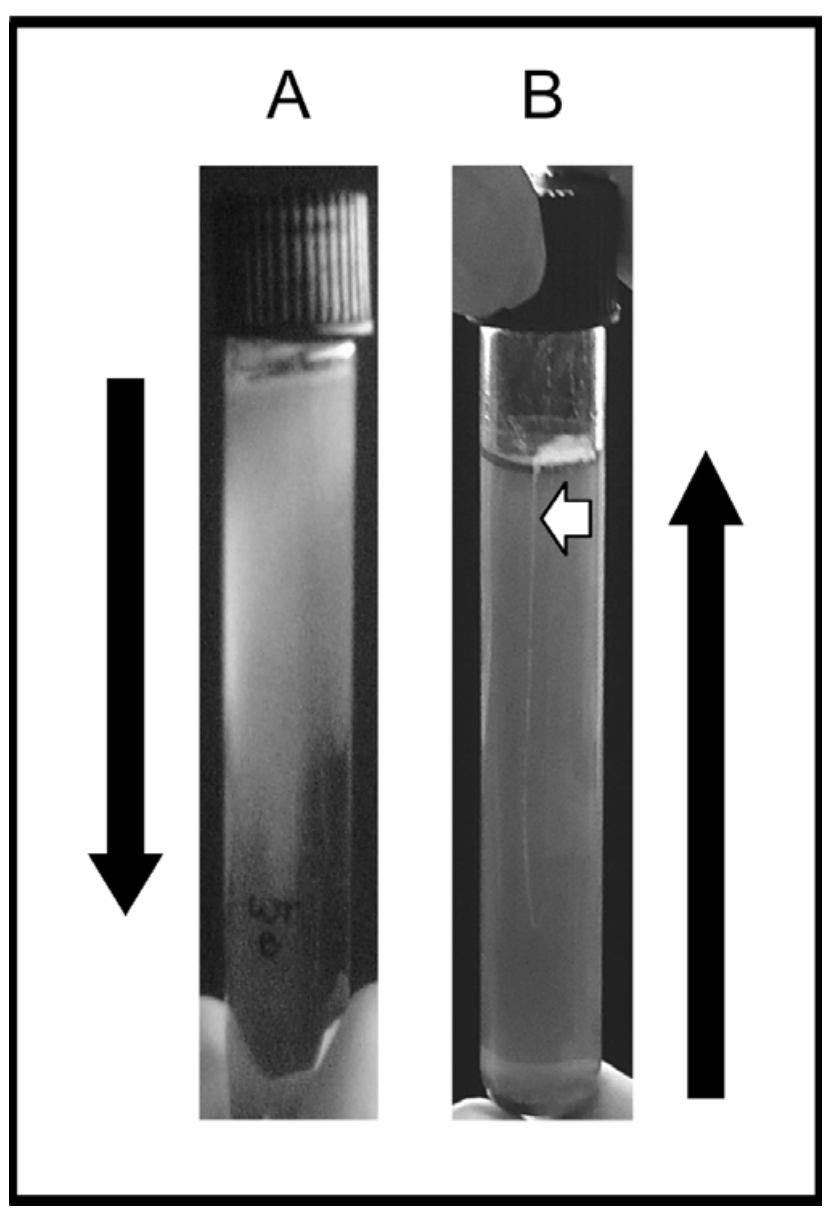

Fig. (1). B. abortus growth under microaerobic conditions. 10-day old cultures grown in semi-solid (A) or liquid (B) media at $37^{\circ} \mathrm{C}$. Bacterial aggregates are visualized at the top of the liquid medium as threads hanging (short arrow) from a discontinuous ring. Note bacterial mass at the bottom of the tube. Large arrows indicate bacterial growth direction. measurement was possible and no surface pellicle was produced. Although B. abortus barely adheres to glass at the airliquid interface, it was unable to grow in biofilms on this abiotic surface, under our experimental conditions. To ensure cell viability, samples of threads were stained with the vital stain Acridine orange and observed by fluorescence microscopy [13]. Almost all the cells stained orange, indicating the threads were composed of adherent living bacteria. These results demonstrate that wild-type $B$. abortus autoaggregates under low oxygen tension and nutrient limitation, acquiring in this form the capacity to move.

One possible explanation for the motility of Brucella could be the involvement of flagella. It is interesting to note that even though B. abortus possesses and expresses flagellar genes, the bacteria have been reported as non-motile in vitro [14]. The inability to synthesize the flagellar hook protein, FlgE, renders a non-flagellated strain. Thus, wild-type $B$. abortus 2308 and the isogenic flgE [15] were examined in liquid and semi-solid media incubated under microaerobiosis. The results showed a wild-type phenotype for the flgE mutant in both types of cultures, eliminating the possibility of flagellar motility. Changes in buoyancy and coordinated population-migration may explain the bacterial behaviour in liquid and in semi-solid media, respectively.

To further analyse the conditions given by the aggregation phenotype, daily samples starting from overnight cultures were taken from the semi-solid and liquid cultures, incubated under aerobic or microaerobic conditions. Samples were examined microscopically and plated to assess colony morphology. Aggregates were only observed from day 3 of incubation under microaerobiosis. Colony morphology derived from aggregates in all cases was the same as that of non-aggregative cells obtained during log or stationary phase under aerobic conditions. Scanning electron microscopy (SEM) of the aggregates revealed an amorphous matrix surrounding the bacteria (Fig. 2A). The SEM observations were conducted at $10 \mathrm{kV}$ in a Philips XL 30 TMP. Each specimen was mounted on an aluminium stub and coated with goldpalladium.

To verify the polysaccharidic nature of the matrix, samples were stained with calcofluor and Congo red. All samples were fixed by addition of $3.7 \%$ formaldehyde solution prior to immobilization. Slides containing the samples were air-dried, then covered with a 2:1 mixture of saturated aqueous Congo red solution and $10 \%$ (vol/vol) Tween 80 or freshly prepared $0.1 \%$ calcofluor (Cellufluor Polyscience Inc., PA, USA) in distilled water. The appearance of positive fluorescence in the aggregates with both dyes but not in isolated cells suggests the presence of $\beta(1,3)$ or $\beta(1,4)$ glucans in the B. abortus extracellular polymeric matrix. Exopolymers such as polysaccharides and fimbria are important components of the extracellular matrix. B. abortus does not express fimbria but produces $\beta(1,2)$ cyclic glucan. To identify whether this glucan, its transporter (Cgt), or the enzyme that adds succinyl residues to the glucan (Cgm) were involved producing the extracellular matrix, the experiments in liquid and semi-solid media were performed with cgs [16], cgt [17], and cgm [18] mutants. Cgs synthesizes this polymer. These strains exhibit wild-type behaviour, indicating that $\beta(1,2)$ cyclic glucan, its transporter, and its modifier do 

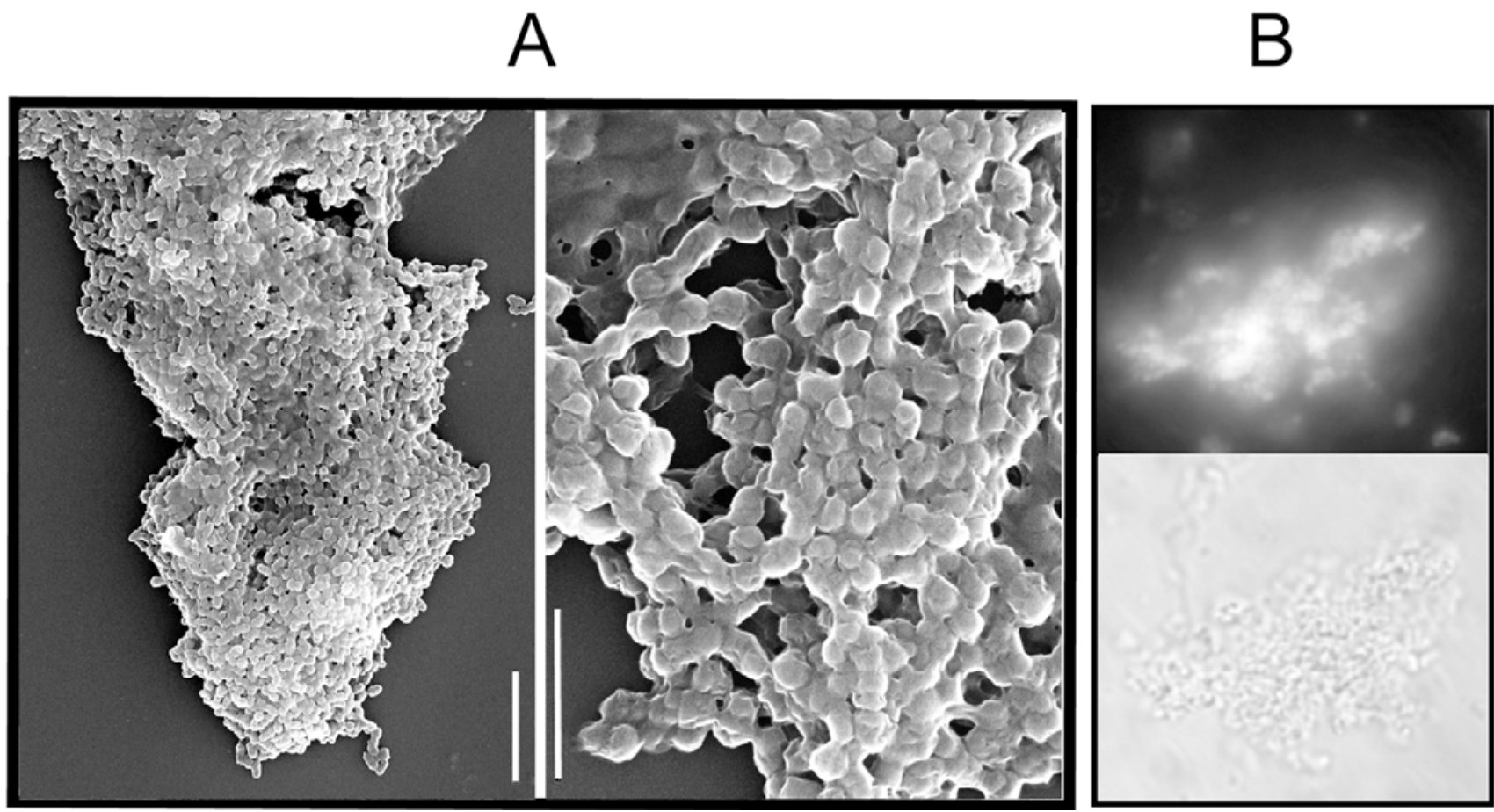

Fig. (2). Microscopic observations of B. abortus aggregation under microaerobic conditions. (A) Scanning electron micrograph of B. abortus 2308; scale bars: $5 \mu \mathrm{m}$ (left) and $2 \mu \mathrm{m}$ (right). (B) Fluorescence (upper) and phase-contrast (lower) micrographs of $B$. abortus vjbR stained with calcofluor. X 100.

not mediate the matrix production needed to adhere or displace. Since LPS also participates in bacterial adherence [19], B. abortus pgm [20] was included in our experiments. This strain, deleted for the gene encoding phosphoglucomutase, does not expose lipopolysaccharide-O antigen on the surface, leading to a rough phenotype. Again, this mutant was able to aggregate and displace, showing that the lipopolysaccharide does not play an important role in the adhesion and movement of $B$. abortus under our experimental conditions. These results also demonstrate that matrix production is possible in rough Brucella strains.

Absence of the VjbR regulator in B. abortus does not lead to the aggregation phenotype observed in the B. melitensis $v j b R$ when grown aerobically [4] and wild-type $B$. abortus aggregates under our experimental conditions; therefore, we generated a knock-out mutation in $v j b R$ to determine its effect on the B. abortus phenotype. Thus, cultures of B. abortus vjbR [21] in liquid and semi-solid media under microaerobic conditions were examined microscopically and compared to wild type. The mutant was able to aggregate (Fig. 2B) and move as the wild type. These results clearly show that the transcriptional regulator VjbR is not involved in the aggregation and displacement of B. abortus under microaerobic conditions. Since it regulates flagellar genes [22], these results also demonstrate that flagella are not involved in the bacterial phenotype under microaerobiosis.

\section{The Desiccation Tolerance of $B$. Abortus}

The extracellular matrix usually protects aggregated cells from different stress conditions or even from the proximity of toxic compounds, thus increasing bacterial survival. It has been reported that B. abortus can survive in materials such as soil, pasture, or manure and that ingestion of these contaminated materials may be one route by which animals or humans in barns acquire brucellosis [23]. Hence, we investigated if the matrix-embedded cells obtained under starvation and oxygen limitation could encourage B. abortus desiccation tolerance. To avoid dealing with the increasing number of dead cells during the stationary phase of growth and taking into account that the matrix was observed at day 3 postincubation under microaerobic conditions, the experiments were performed with 3-day old cultures grown in BB under aerobic or microaerobic conditions. First, the amount of Congo red bound to cells from each culture was quantified [24] by dissolving the cell-bound stain in DMSO and reading the OD of the supernatant at $490 \mathrm{~nm}$. Standard preparations of the stain in DMSO were made to generate a standard curve of $\mathrm{mg}$ Congo red $/ \mathrm{mL}$ and viable cells were determined by plating serial dilutions of each culture. As shown in Fig. (3), the amount of stained matrix per cell in cells grown under microaerobic conditions was 4 times higher than that observed under aerobic conditions. After observing this significant difference in the extracellular material, small triplicate samples were tested for desiccation tolerance [25]. Briefly, 20- $\mu \mathrm{L}$ samples were taken in triplicate. A total of $480 \mu \mathrm{L}$ media was immediately added to one of the samples and serial dilutions were plated on BB agar for bacterial counts. The second sample was allowed to dry at $30^{\circ} \mathrm{C}$, while the third sample remained unexposed for the same period of time as a control. After total desiccation, 500 or $480 \mu \mathrm{L}$ media was added and the cells recovered to estimate percent survival. These results (Fig. 3) show that cells provided with an extracellular matrix are better able to survive desiccation than planktonic cells under our experimental conditions. Although extracellular matrix usually protects 


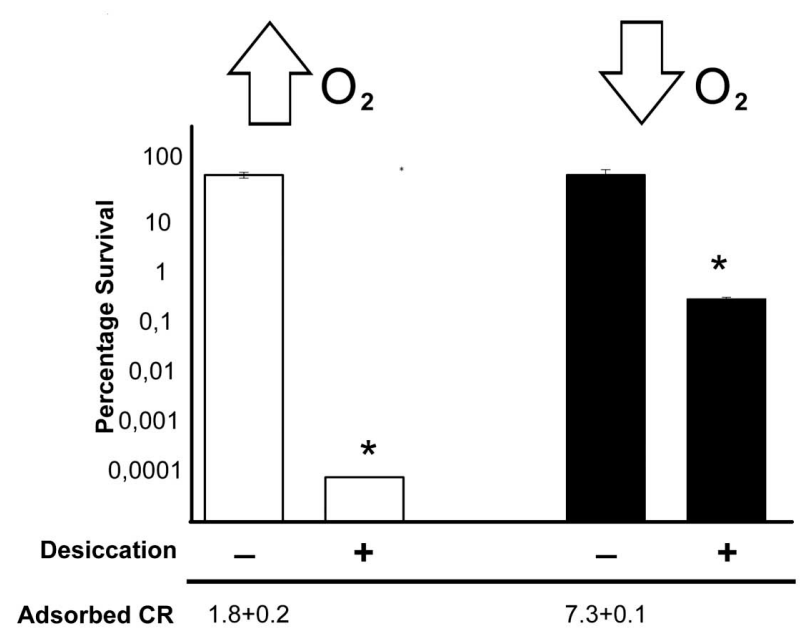

Fig. (3). Desiccation tolerance of B. abortus related to its matrix production under aerobic (white bars) or microaerobic (black bars) conditions. Bacterial matrix was stained with Congo red (CR) and expressed as $\mu \mathrm{g}$ of stain/CFU. * indicates $p<0.005$.

cells from desiccation, further studies will be needed to identify the components of the extracellular matrix and its participation in survival of $B$. abortus in the environment.

\section{Biofilm Development of $B$. Abortus}

To investigate B. abortus biofilm formation on other abiotic surfaces such as plastic, inocula from cultures containing about $10^{8} \mathrm{CFU} / \mathrm{mL}$ were diluted 1:100 into $2 \mathrm{~mL} \mathrm{BB}$ per well of polyvinyl-chloride 24-well plates (Falcon, Becton Dickinson, France) and into $30 \mathrm{~mL}$ of BB in polystyrenetreated $60-\mathrm{mL}$ flasks (Corning Inc., New York, USA). The cells showed very little adherence to the polyvinyl-chloride plates in a microaerobic environment generated by a $5 \% \mathrm{CO}_{2}$ incubator for a period of 20 days. However, after the same period of time, biofilms appeared on the sides of the polystyrene flasks. This type of adherence was more stable than that observed on glass or plates since it could withstand the staining procedure. No surface pellicle was observed. To differentiate between attachment of aggregative cells and biofilm growth, 2-day-old B. abortus cultures grown in polystyrene flasks were maintained at $37^{\circ} \mathrm{C}$ for longer under conditions that allow (microaerobiosis) or do not allow (anaerobiosis) bacterial replication. Anaerobiosis was achieved using an AnaeroGen ${ }^{\mathrm{TM}}$ sachet (Oxoid, Hampshire, UK) to generate less than $1 \% \mathrm{O}_{2}$ and $9-13 \% \mathrm{CO}_{2}$ in a sealed jar. From days 9 to 11 , adherence on the sides of the flasks was detected at the air-liquid interface in both conditions. At the end of the experiment, when bacterial attachment was detected by crystalviolet staining, we observed a blue line at the air-liquid interface in cultures maintained under anaerobiosis. In contrast, the sides of the flasks maintained under low oxygen tension were completely stained (Fig. 4). These results indicate that aggregative cells were able to float and adhere under both conditions but the biofilm was the consequence of downward growth from the attached cells. No surface pellicle was observed. The inability to develop biofilms in plates could be attributed to the components of the plastic. Interestingly,

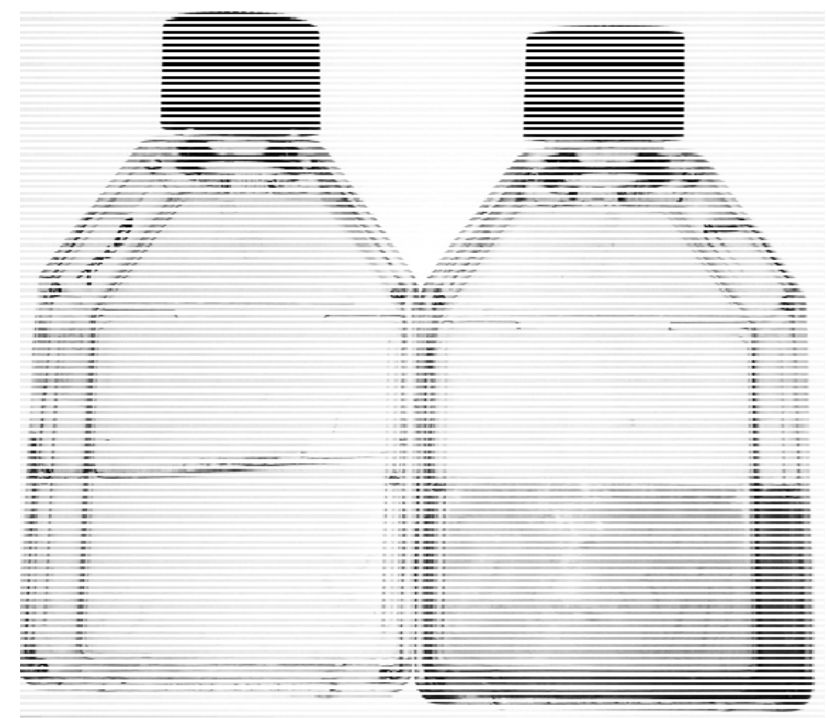

Fig. (4). B. abortus biofilm development on polystyrene flasks. Crystal-violet stained flasks from cultures grown under anaerobic (left) or microaerobic (right) conditions at $37^{\circ} \mathrm{C}$ during 18 days after saturation.

the matrix-embedded cells inside the flask found a better attachment with the pre-treated sides than the others, and this condition promoted biofilm production.

Bacteria in biofilms are not only resistant to desiccation but are also protected from interaction with substances such as antibiotics, antibodies, and disinfectants. Therefore, considering the epidemiology and chronicity of brucellosis, the characteristics of $B$. abortus under these conditions must be analysed at the genetic level to support development of eradication programs. Studies are underway to identify the components of the extracellular matrix and the genes involved in their synthesis and transport.

\section{CONCLUSION}

The results presented here demonstrate that starved $B$. abortus grown under microaerobiosis produce extracellular matrix, autoaggregate, move, and adhere to abiotic surfaces without the involvement of $\mathrm{VjbR}$, the lipopolysaccharide-O antigen, cyclic $\beta(1,2)$ glucan, or flagella. Matrix-producing cells are more resistant to desiccation and promote the development of biofilms.

\section{CONFLICT OF INTEREST}

The authors confirm that this article content has no conflicts of interest.

\section{ACKNOWLEDGEMENTS}

B. abortus mutant strains were kindly provided by R.A.Ugalde. This work was supported by grants from the National Agency for Science and Technology PICT-0651 and PIP-5463 from the National Research Council of Argentina (CONICET). M.A., M.S.R. and N.S. are members of the Research Career, CONICET. 


\section{REFERENCES}

[1] Hall-Stoodleyl L, Stoodley P. Evolving concepts in biofilm infections. Cell Microbiol 2009; 11: 1034-43.

[2] Burmolle M, Thomsen TR, Fazli M, et al. Biofilms in chronic infections - a matter of opportunity - monospecies biofilms in multispecies infections. FEMS Immunol Med Microbiol 2010; 59: 32436.

[3] Labbate M, Zhu H, Thung L, et al. Quorum-sensing regulation of adhesion in Serratia marcescens MG1 is surface dependent. J Bacteriol 2007; 189: 2702-11.

[4] Uzureau S, Godefroid M, Deschamps C, Lemaire J, De Bolle X, Letesson JJ. Mutations of the quorum sensing-dependent regulator VjbR lead to drastic surface modifications in Brucella melitensis. $\mathrm{J}$ Bacteriol 2007; 189: 6035-47.

[5] Wang Y, Chen Z, Qiao F, et al. The type IV secretion system affects the expression of Omp25/Omp31 and the outer membrane properties of Brucella melitensis. FEMS Microbiol Lett 2010; 303: 92100 .

[6] Godefroid M, Svensson MV, Cambier P, et al. Brucella melitensis $16 \mathrm{M}$ produces a mannan and other extracellular matrix components typical of a biofilm. FEMS Immunol Med Microbiol 2010; 59: 364-77.

[7] Kohler S, Porte F, Jubier-Maurin V, Ouahrani-Bettache S, Teyssier J, Liautard JP. The intramacrophagic environment of Brucella suis and bacterial response. Vet Microbiol 2002; 90: 299-309.

[8] Roop RM 2nd, Gaines JM, Anderson ES, Caswell CC, Martin DW. Survival of the fittest: how Brucella strains adapt to their intracellular niche in the host. Med Microbiol Immunol 2009; 198: 221-38.

[9] Taminiaub B, Daykin M, Swift S, et al. Identification of a quorumsensing signal molecule in the facultative intracellular pathogen Brucella melitensis. Infect Immun 2002; 70: 3004-11.

[10] Weeks JN, Galindo CL, Drake KL, Adams GL, Garner HR, Ficht TA. Brucella melitensis VjbR and C12-HSL regulons: contributions of the N-dodecanoyl homoserine lactone signaling molecule and LuxR homologue VjbR to gene expression. BMC Microbiol 2010; 10: 167.

[11] Uzureaus S, Lemaire J, Delaive E, et al. Global analysis of quorum sensing targets in the intracellular pathogen Brucella melitensis 16 M. J Proteome Res 2010; 9: 3200-17.

[12] O'Toole GA, Kolter R. Initiation of biofilm formation in Pseudomonas fluorescens WCS365 proceeds via multiple, convergent signalling pathways: a genetic analysis. Mol Microbiol 1998; 28: 44961.

[13] Zambrano MM, Siegele DA, Almiron M, Tormo A, Kolter R. Microbial competition: Escherichia coli mutants that take over stationary phase cultures. Science 1993; 259: 1757-60.
[14] Fretin D, Fauconnier A, Kohler S, et al. The sheathed flagellum of Brucella melitensis is involved in persistence in a murine model of infection. Cell Microbiol 2005; 7: 687-98.

[15] Marchesini MI. Búsqueda e identificación de proteínas secretadas por Brucella abortus: caracterización de un sustrato del sistema VirB. PhD Thesis. San Martín: Universidad de San Martín 2009.

[16] Briones G, Inon de Iannino N, Roset M, Vigliocco A, Paulo PS, Ugalde RA. Brucella abortus cyclic beta-1,2-glucan mutants have reduced virulence in mice and are defective in intracellular replication in HeLa cells. Infect Immun 2001; 69: 4528-35.

[17] Roset MS, Ciocchini AE, Ugalde RA, Inon de Iannino N. Molecular cloning and characterization of cgt, the Brucella abortus cyclic beta-1,2-glucan transporter gene, and its role in virulence. Infect Immun 2004; 72: 2263-71.

[18] Roset MS, Ciocchini AE, Ugalde RA, Inon de Iannino N. The Brucella abortus cyclic beta-1,2-glucan virulence factor is substituted with O-ester-linked succinyl residues. J Bacteriol 2006; 188: 5003-13.

[19] Zaidi TS, Fleiszig SM, Preston MJ, Goldberg JB, Pier GB. Lipopolysaccharide outer core is a ligand for corneal cell binding and ingestion of Pseudomonas aeruginosa. Invest Ophthalmol Vis Sci 1996; 37: 976-86.

[20] Ugalde JE, Czibener C, Feldman MF, Ugalde RA. Identification and characterization of the Brucella abortus phosphoglucomutase gene: role of lipopolysaccharide in virulence and intracellular multiplication. Infect Immun 2000; 68: 5716-23.

[21] Arocena GM, Sieira R, Comerci DJ, Ugalde RA. Identification of the quorum-sensing target DNA sequence and N-Acyl homoserine lactone responsiveness of the Brucella abortus virB promoter. J Bacteriol 2010; 192: 3434-40.

[22] Delruem M, Deschamps C, Leonard S, et al. A quorum-sensing regulator controls expression of both the type IV secretion system and the flagellar apparatus of Brucella melitensis. Cell Microbiol 2005; 7: 1151-61.

[23] Corbel MJ. Brucellosis in humans and animals. World Health Organization, WHO/CDS/EPR/2006.7. Geneva, Switzerland : WHO Press 2006

[24] Rhodes ER, Shoemaker CJ, Menke SM, Edelmann RE, Actis LA. Evaluation of different iron sources and their influence in biofilm formation by the dental pathogen Actinobacillus actinomycetemcomitans. J Med Microbiol 2007; 56: 119-28.

[25] Gualdi L, Tagliabue L, Bertagnoli S, Ierano T, De Castro C, Landini P. Cellulose modulates biofilm formation by counteracting curlimediated colonization of solid surfaces in Escherichia coli. Microbiology 2008; 154: 2017-24.

(C) Almiron et al.; Licensee Bentham Open.

This is an open access article licensed under the terms of the Creative Commons Attribution Non-Commercial License (http://creativecommons.org/licenses/ by-nc/3.0/) which permits unrestricted, non-commercial use, distribution and reproduction in any medium, provided the work is properly cited. 